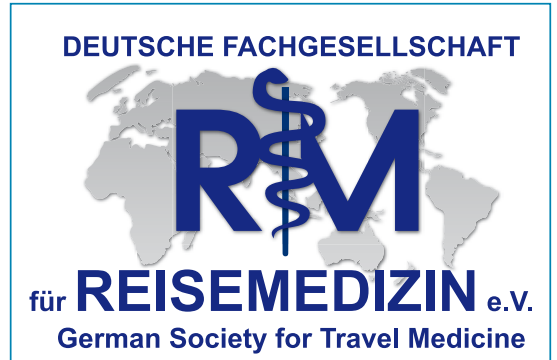

Hansaallee 299, 40549 Düsseldorf

Tel.: 0211/5202581 Fax: 0211/5202583

E-Mail: info@fachgesellschaft-reisemedizin.de www.fachgesellschaft-reisemedizin.de

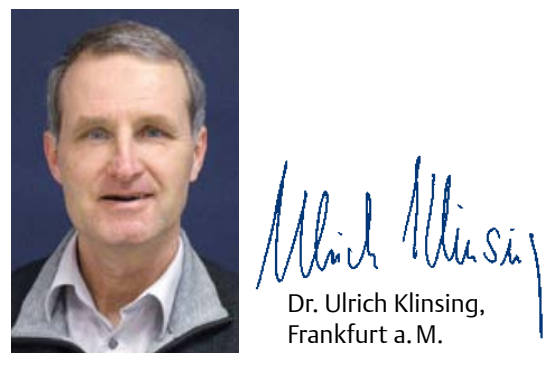

\title{
Liebe Mitglieder der Deutschen Fachgesellschaft für Reisemedizin,
}

vor über einem Jahr haben wir die Umbenennung von „Fachverband“ in „Fachgesellschaft" vollzogen. Wir wollten damit unter anderem unserem Anspruch Ausdruck verleihen, neben einer reinen Verbandstätigkeit auch im wissenschaftlichen Bereich reisemedizinische Inhalte zu transportieren und zu vertreten. Seither haben wir zum einen alle bisherigen Aufgaben mit unveränderten Anstrengungen weiter verfolgt, uns aber zum anderen zusätzlich vermehrt um den reisemedizinisch-wissenschaftlichen Bereich gekümmert.

Besonders der Fachausschuss „Qualitätssicherung" unter der Leitung von Dr. Rosemarie Mazzola hat sich dieser Aufgabe gewidmet. Der Fachausschuss baut zurzeit Kontakte zu Universitäten auf, um wissenschaftliche Arbeiten und Dissertationen mit reisemedizinischen Fragestellungen zu fördern und hierdurch dazu beizutragen, unser reisemedizinisches Tagesgeschäft auf eine wissenschaftlich fundiertere Grundlage zu stellen. Auf Initiative von Prof. Günter Schmolz, ebenfalls aus diesem Fachausschuss heraus, konnten Kontakte zur Apollon Hochschule der Gesundheitswirtschaft in Bremen geknüpft und mit dem Abschluss einer Kooperationsvereinbarung vertieft werden. Mehr Informationen dazu finden Sie auf der Folgeseite.

Dies ist insbesondere deshalb bemerkenswert, da hierdurch reisemedizinische Inhalte an akademische Berufsgruppen vermittelt werden, die als Nichtmediziner federführend im Tourismus tätig sind. In einer gemeinsamen Presseerklärung betont die Dekanin der Universität, Prof. Viviane Scherenberg, dass reisemedizinisches Wissen für angehende Manager im Bereich Gesundheitstouristik notwendiger denn je sei. Wir freuen uns, dass die Apollon Hochschule diesen wichtigen Bedarf erkannt hat und die DFR einen Beitrag zur Qualifizierung der Studierenden und Absolventen des Bachelor-Studiengangs Gesundheitstourismus im Hinblick auf reisemedizinische Themen leisten kann. Hierdurch könnte ein Brückenschlag in die Wege geleitet werden, der uns bereits seit vielen Jahren am Herzen liegt, in dem wir aber nie so richtig voran gekommen sind.

Nochmals einladen möchte ich Sie zur Teilnahme an unserer Jahrestagung am 21. und 22. September in Weimar. Das wissenschaftliche Programm ist wie gewohnt facettenreich und so zusammengestellt, dass es große Teile der Reisemedizin abdeckt. Es wird sicher auch dieses Mal den nach den erfolgreichen Tagungen der letzten Jahre hohen Ansprüchen der Teilnehmer gerecht werden. Wir möchten mit der Veranstaltung den Rahmen für einen Austausch unter reisemedizinischen Experten schaffen, als die wir unsere Mitglieder ansehen. Besonders willkommen sind uns selbstverständlich auch die Mitglieder aller anderen in dieser Zeitschrift vertretenen Fachgesellschaften, zumal die Möglichkeit eines Blicks über den Tellerrand immer ein wesentlicher Aspekt unserer Tagungen ist. Unter dem Programmpunkt „knifflige reisemedizinischen Beratungsfälle“ haben Sie die Möglichkeit, interessante Fälle aus ihrer reisemedizinischen Praxis im Plenum zur Diskussion zu stellen. Wir möchten Sie dazu anregen und ermutigen, sich hier zu beteiligen. Gerne können Sie das Angebot von Rosemarie Mazzola in Anspruch nehmen, Ihren Fall bereits im Vorfeld der Tagung aufzubereiten.

Ganz besonders hinweisen möchte ich auf die Mitgliederversammlung der DFR am späten Nachmittag des ersten Veranstaltungstags. Es werden für die weitere Entwicklung unserer Fachgesellschaft richtungsweisende Beschlüsse zu fassen sein. Dies betrifft neben Entscheidungen zu unserer Geschäftsstelle und zur zukünftigen Vereinsführung auch die Höhe des Jahresbeitrags, der, wie im Rahmen der letzten Versammlung von unseren Mitgliedern angeregt, den gemeinsam formulierten Zielen entsprechend angepasst werden soll. Über ein zahlreiches Erscheinen von Ihnen, unseren Mitgliedern, wird sich der gesamte Vorstand sehr freuen.

In der Gewissheit, einen großen Teil von Ihnen wieder bei der Jahrestagung begrüßen zu dürfen, verbleibe ich

Ihr Ulrich Klinsing 


\title{
Kooperation mit der Apollon Hochschule
}

\author{
Letter of Intent \\ zwischen \\ der Deutsche Fachgesellschaft für \\ Reisemedizin e. V., vertreten durch den Vorsitzenden, Dr. med. Ulrich Klinsing,

$$
\text { - im Folgenden „Deutsche Fachgesellschaft für Reisemedizin“ und }
$$ \\ der APOLLON Hochschule der Gesundheitswirtschaft $\mathrm{GmbH}$, vertreten durch die \\ Geschäftsführer Heinrich Dieckmann und Michael Timm, Universitätsallee 18, \\ 28359 Bremen
}

- im Folgenden „APOLLON Hochule“ -

Die Apollon Hochschule der Gesundheitswirtschaft gehört zur Klett-Verlagsgruppe in Stuttgart. Sie wurde im Jahr 2005 als Fernhochschule, University of Applied Sciences, gegründet und hat ihren Standort in Bremen. Dort finden auch die Präsenzseminare statt. Die Hochschule hat sich im Bereich Gesundheitswirtschaft sehr gut etabliert und einige interessan- te Bachelor- und Master-Studiengänge entwickelt, unter anderem den Bachelor Gesundheitstourismus (B.A.). Die Studiengänge sind berufsbegleitend und praxisorientiert konzipiert, der Studienbeginn ist individuell ganzjährig möglich. Für den B.A. Gesundheitstourismus haben verschiedene (Vorstands-)Mitglieder der DFR in den letzten Monaten Studi- enhefte entwickelt, in denen die ganze Breite der Reisemedizin für Touristikmanager abgebildet werden soll. Die Absolventen dieses Studiengangs sollen mit ihrem (reise-)medizinischen Grundwissen kompetente Gesprächspartner der niedergelassenen Ärzteschaft und der Reisenden sein. Der Studiengang richtet sich an eine breite Zielgruppe aus den Berufsfeldern Ökonomie, Tourismus und Gesundheit: unter anderem an Touristikkaufleute, nichtärztlich Beschäftigte in der Gesundheitsförderung und Rehabilitation, sowie an kaufmännische Mitarbeiter von Kur- und Bädereinrichtungen. Die Verbindung zwischen DFR und Apollon Hochschule hat ihren ersten Niederschlag in diesem „Letter of Intent“ gefunden.

Die Hochschule hat diesen Einstieg in eine Kooperation mit einer Pressemitteilung bekannt gemacht.

Prof. Dr. Günter Schmolz, Stuttgart

\section{Liebe Kolleginnen und Kollegen,}

auch die 15. Jahrestagung der DFR in Weimar wird unter der wissenschaftlichen Leitung von Dr. Tomas Jelinek mit der Diskussion von Kasuistiken beginnen. Diese Eröffnung der Jahrestagung hat sich bewährt und findet bei den Teilnehmern eine positive Resonanz.

Zur abwechslungsreichen Gestaltung dieses Programmpunkts benötige ich jedoch Ihre Mitarbeit: Wenn Sie in Ihrer reisemedizinischen Tätigkeit ob Praxis, Gesundheitsamt, in der Betriebs- oder Arbeitsmedizin - einen interessanten Beratungsfall haben oder hatten, möchte ich Sie bitten, den Fall im Rahmen der Jahrestagung vorzustellen.

Sie können mir die Daten zur Vorstellung auch übergeben, wenn Sie die Vorstellung der Kasuistik nicht selbst übernehmen möchten oder nicht anwesend sein können. Ich möchte Sie zur Mitwirkung auffordern und ermutigen: Ihre Fälle aus der Praxis bereichern die Tagung.

Bitte geben Sie mir 2 Wochen vor der Tagung über die Geschäftsstelle Bescheid, wenn Sie mit einer Kasuistik einen Beitrag leisten können.

Mit der Hoffnung auf eine zahlreiche Beteiligung und herzlichen Grüßen

Dr. Rosemarie Mazzola, Freiburg

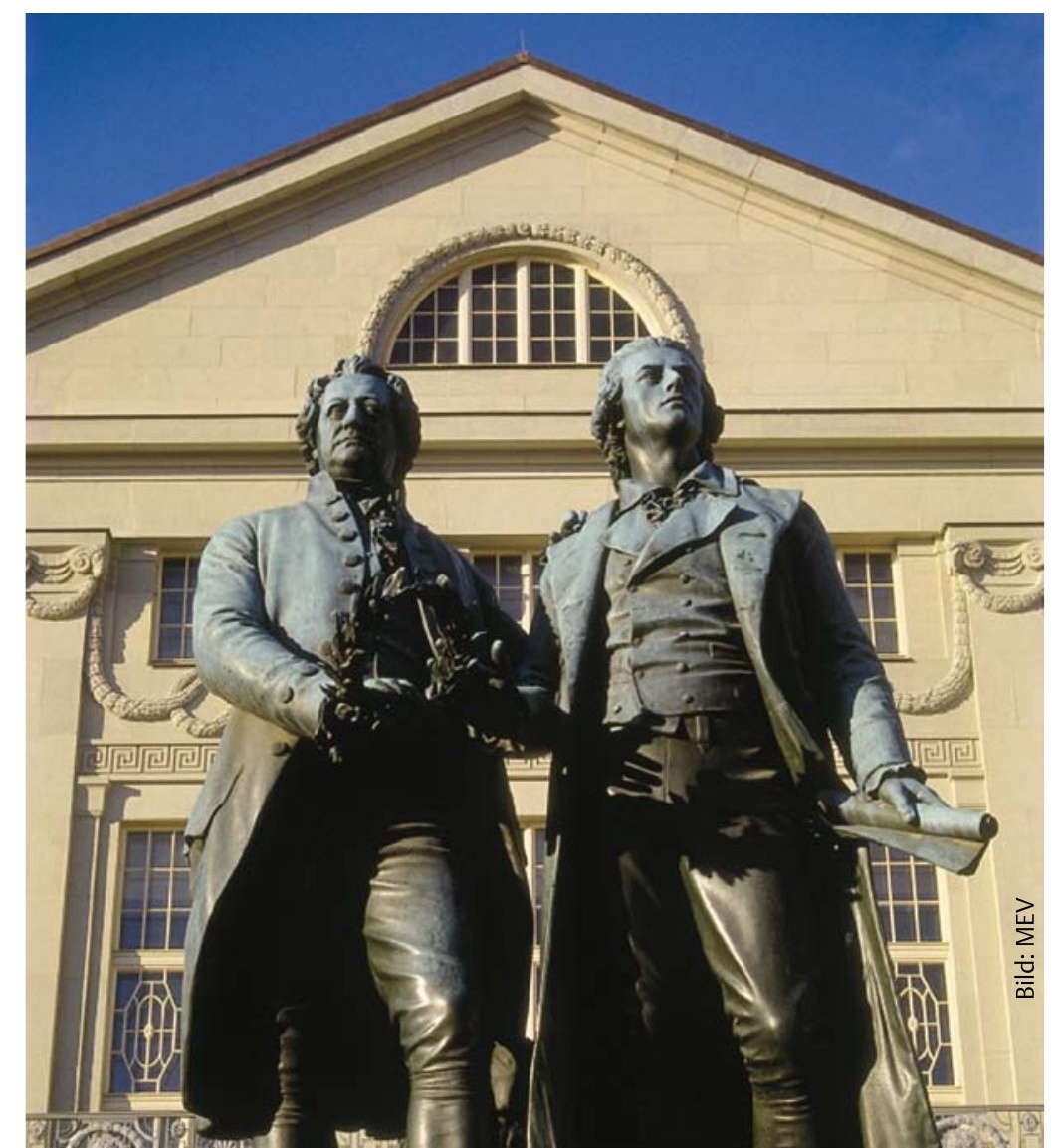




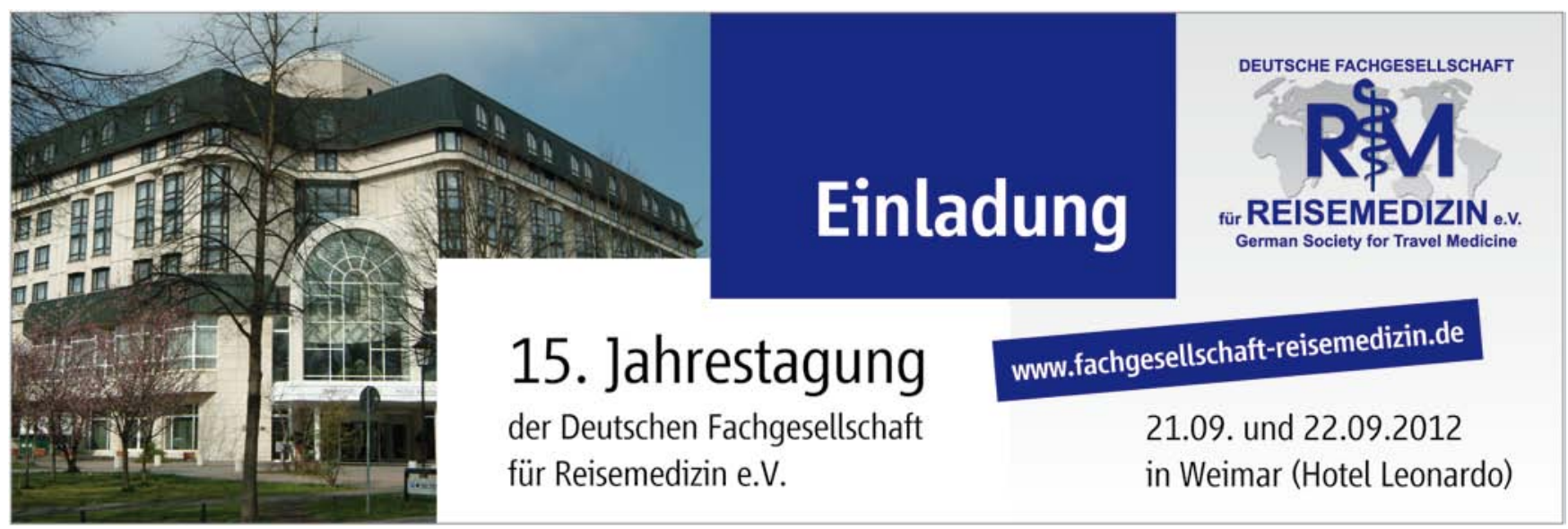

Programm

Freitag, 21. September

12:30 Uhr Begrüßungsimbiss

13:00-13:15 Uhr Begrüßung und Eröffnung

der 15. Jahrestagung

Dr. Ulrich Klinsing, Frankfurt

Update Reisemedizin

Vorsitz:

Dr. Ulrich Klinsing, Frankfurt

13:15-14:15 Uhr Knifflige reisemedizinische Beratungsfälle Dr. Rosemarie Mazzola, Freiburg

14:15-15:15 Uhr Malaria: aktuelle Trends Dr. Klaus-J. Volkmer, Buchholz

15:15-15:45 Uhr Kaffeepause

15:45-16:30 Uhr Impfungen bei Immunsuppression: der Weg zu einem Europäischen Konsensus PD Dr. Tomas Jelinek, Düsseldorf

16:45 Uhr Mitgliederversammlung

18:30 Uhr
Stadtrundgang ab Tagungshotel anschließend gemeinsames Abendessen
Samstag, 22. September

Klinischer Schwerpunkt

Vorsitz: $\quad$ Dr. Stefan Eßer, Neu-Isenburg

9:00-9:45 Uhr Fallbeispiele Reiserückkehrer Prof. Dr. Thomas Weinke, Potsdam

9:45-10:30 Uhr Fieber bei Tropenrückkehr Dr. Dr. Martin Haditsch, Hannover

10:30-11:00 Uhr Kaffeepause

11:00-11:45 Uhr Fallbeispiele vom CRM-Konsiltelefon Dr. Ute Schwarz, Berlin

11:45-12:30 Uhr Medizinische Aspekte der Beratung bei Freiwilligendiensten Dr. Luise Prüfer-Krämer, Bielefeld

12:30-13:30 Uhr Mittagspause

Aktuelle Themen Vorsitz:

Prof. Dr. Günter Schmolz, Stuttgart

\begin{tabular}{|c|c|}
\hline 13:30-14:15 Uhr & $\begin{array}{l}\text { Wilderness Medicine } \\
\text { Dr. Fritz Holst, Marburg }\end{array}$ \\
\hline 14:15-15:00 Uhr & $\begin{array}{l}\text { Medizin auf dem Wasser: } \\
\text { Expeditionen und Kreuzfahrten } \\
\text { Dr. Peter Stein, Frankfurt }\end{array}$ \\
\hline 15:00-15:30 Uhr & Kaffeepause \\
\hline 15:30-16:15 Uhr & $\begin{array}{l}\text { Reisemedizin in Europa: ein Vergleich } \\
\text { PD Dr. Tomas Jelinek, Düsseldorf }\end{array}$ \\
\hline 16:15-17:00 Uhr & $\begin{array}{l}\text { Ärztlich begleitetes Reisen: } \\
\text { Qualitätsstandards } \\
\text { Dr. Burkhard Rieke, Düsseldorf }\end{array}$ \\
\hline
\end{tabular}

Veranstaltungsort: Hotel Leonardo, Belvederer Allee 25, 99425 Weimar

Die Zertifizierung der Veranstaltung wird bei der zuständigen Landesärztekammer beantragt.

Information und Anmeldung:

www.fachgesellschaft-reisemedizin.de,

Deutsche Fachgesellschaft für Reisemedizin e.V.,

Hansaallee 299,

40549 Düsseldorf,

Tel: 0211-520 25 81, Fax: 0211-520 2583 